\title{
El doctorado en educación de la Universidad Santo Tomás. Cinco años de registro calificado ${ }^{1}$
}

\author{
José Duván Marín Gallego²
}

Fecha de recepción: 26 de marzo de 2015

Fecha de revisión: 11 de abril de 2015

Fecha de aprobación: 9 de mayo de 2015

\section{Resumen}

Este artículo corresponde a una reflexión y, al mismo tiempo, a una semblanza de lo que ha sido y lo que ha hecho el Doctorado en Educación de la Universidad Santo Tomás al cumplirse los primeros cinco años del registro calificado, otorgado por el Ministerio de Educación Nacional. Se resumen los principales hechos y acontecimientos que han tenido lugar al interior del programa y en su relación con la comunidad educativa y la sociedad, considerando particularmente su desarrollo, sus logros y avances, así como sus expectativas para los años venideros.

\section{Palabras clave:}

Doctorado en Educación, registro calificado, seminarios, investigación, tesis.

1 Documento de reflexión.

2 Doctor en Educación, con estudios posdoctorales en Ciencia y Narrativa, magister en Evaluación de la Educación, especialista en Docencia Universitaria, abogado y licenciado en Filosofía. Actualmente docente del Doctorado en Educación de la Universidad Santo Tomás; coordinador de la "Línea de Currículo y Evaluación" y líder del grupo "Investigación Educativa", categoría D de Colciencias. Carrera 9A No.63-28. Bogotá, Colombia. Correo electrónico: joseduvanmarin@ustadistancia.edu.co 


\section{Ph.D. in education at Universidad Santo Tomas. Five years of official registration}

\section{Abstract}

This article is a reflection and a semblance of what has been and has done the Doctorate in Education at the University of Santo Tomas in the first five years of Registration Qualified, by the Ministry of National Education. It summarizes the main facts and events that have occurred within the program and their relationship with the educational community and society, particularly considering their development, achievements, advances and expectations for future years.

\section{Keywords:}

Ph.D., qualified registry, seminars, research, thesis.

\section{O Doutorado em Educação da Universidade Santo Tomás. Cinco anos de registro qualificado.}

\section{Resumo}

Este artigo é uma reflexão e, ao mesmo tempo, para uma aparência de que foi e do que ele fez doutorado em Educação pela Universidade de St. Thomas após a conclusão dos primeiros cinco anos de registro qualificada, emitido pelo Ministério da Educação nacional. os principais fatos e acontecimentos que tiveram lugar dentro do programa e sua relação com a comunidade educativa e da sociedade, especialmente considerando seu desenvolvimento, suas conquistas e avanços, bem como as suas expectativas para os próximos anos são resumidas. 


\section{Palavras-chave:}

Ed.D., registro qualificada, seminários, pesquisa, tese.

\section{Introducción}

El 26 de febrero del presente año se cumplieron los primeros cinco años del registro calificado del Doctorado en Educación de la Universidad Santo Tomás. Cinco años hace cuando el rector de la Universidad, en ese entonces el Padre José Antonio Balaguera O.P., invitaba a un grupo de funcionarios y docentes a su despacho para dar la noticia y escuchar la lectura de la Resolución 1207 del 26 de febrero de 2010, mediante la cual el Ministerio de Educación Nacional le comunicaba a la Universidad que, de acuerdo con el Artículo primero de la parte resolutiva, otorgaba por el término de siete (7) años, el registro calificado al siguiente programa. Institución: Universidad Santo Tomás. Programa: Doctorado en Educación. Sede del programa: Bogotá D. C. Metodología: presencial. Título a otorgar: doctor en Educación. Número de créditos académicos: 80. Firma: el viceministro de Educación Superior.

Con honor me ha correspondido hacer esta semblanza ante la comunidad académica y administrativa del Doctorado en Educación, toda vez que tuve el privilegio de haber contribuido a su gestación, de haberlo visto nacer y de permanecer todavía en el programa en calidad de docente, coordinador de una línea de investigación y director de tesis doctorales.

Cinco años parece mucho para la vida de una institución, pero todavía es muy poco. Se han hecho algunas cosas, pero es más lo que falta por hacer. Sin embargo, ya no es tan importante lo que se hizo, pues lo hecho, hecho está, bien o mal, sino la proyección de lo que se debe hacer de ahora en adelante.

Permítaseme, sin embargo, evocar los principales logros y recordar algunos progresos del doctorado en estos cinco primeros años de su registro calificado.

\section{Desarrollo}

Este Doctorado en Educación que acababa de comenzar su vida jurídica y social, tuvo la fortuna de haber sido el primer programa de doctorado de la Universidad Santo Tomás después de 150 años, como lo recordaba el Provincial de los 
Dominicos, Fray Orlando Rueda O.P.; pero también fue el primer Doctorado en Educación de una institución privada en el país, ya que hasta ese momento solamente funcionaban los doctorados de instituciones del Estado, como el de la Universidad de Antioquia, el más antiguo del país, el de la Red Rudecolombia, conformada por nueve universidades públicas y el institucional de tres universidades: Pedagógica Nacional, Distrital Francisco José de Caldas y la Universidad del Valle.

El nacimiento del Doctorado en Educación fue el resultado de un proyecto de investigación y construcción que duró casi tres años, en el que un equipo conformado por el vicerrector de la VUAD en ese momento, Padre Luis Francisco Sastoque Poveda O.P., el decano de Educación, doctor Francisco Chica Cañas y José Duván Marín G., como docente de la Maestría en Educación. Este proyecto buscaba dar respuesta a las necesidades de desarrollo del país en Ciencia, Tecnología e Innovación, y a los planteamientos del Plan Estratégico de Colciencias en relación con la urgente necesidad de formar investigadores de calidad en los diversos campos de las ciencias, entre las que también se contaban, la educación y la pedagogía.

Para ello, era necesario contar con la experiencia y la madurez académica e investigativa de la Facultad de Educación de la Universidad Santo Tomás que, con más de 35 años de experiencia y de actividad académica, había formado docentes en todos los rincones de la geografía colombiana, no solamente en programas de pregrado a distancia, sino también por medio de las especializaciones, particularmente de Docencia Universitaria y Evaluación de la Educación. Pero especialmente, el doctorado debía soportarse en las maestrías que habían pasado por la Facultad, como la Maestría en Administración Educativa, Maestría en Evaluación de la Educación, Maestría en Investigación y Docencia, Maestría en Educación y Filosofía Latinoamericana que, desde 1980 hasta el año 2000, habían sido muy exitosas como programas de formación en el nivel de posgrados; asimismo, se consideraba especialmente el apoyo inmediato de la actual Maestría en Educación que, desde 2004, ostentaba el registro calificado.

Por otra parte, desde las políticas nacionales se tuvo en cuenta: a) el Plan Nacional de Desarrollo del Gobierno de turno, que se proponía, entre una de las cinco líneas de desarrollo del país, la de "Educación, Cultura e Instituciones"; b) el Plan Sectorial, del Ministerio de Educación Nacional, que buscaba, a la vez, impulsar la formación de docentes e investigadores en maestrías y doctorados, y c) el Plan Decenal de Educación 2006-2016, que recomendaba el fortalecimiento e implementación de la formación de alto nivel (maestrías, doctorados y posdoctorados) 
con pertinencia regional, nacional e internacional y con proyección laboral en todas las áreas del conocimiento, enfatizando en el campo educativo.

Por lo tanto, estaban dadas las condiciones favorables desde las políticas nacionales, legales e institucionales para la creación del programa de Doctorado en Educación de la Universidad Santo Tomás, con un enfoque basado en el humanismo cristiano y social, inspirado en la doctrina de Tomás de Aquino y en el ideario de la Comunidad Dominicana, formadora de generaciones de profesionales, comprometidos con la transformación de la persona y el desarrollo social del país. Este humanismo cristiano tomista no es un humanismo excluyente, por cuanto dialoga con todos los demás humanismos y está abierto a toda concepción del hombre, cualquiera sea su visión que, de acuerdo con una cita del Padre Enrique Almeida, O.P., tomada del Documento Marco del doctorado (2009):

No cabe duda. En las obras de Santo Tomás encontramos los principios fundamentales de aquel humanismo cristiano, que se identifica con la civilización de la persona, la única civilización de la persona verdaderamente humana. Ella tiene por centro al hombre.

Consiste en la máxima realización de la persona como ser inteligente y libre. En la doctrina tomista la persona es el mayor valor de todo el universo visible. Todo se ordena a su bienestar y desarrollo. El hombre tiene significado en la historia, en el trabajo, en la economía, en el progreso y no viceversa. Es el artífice de su destino, el protagonista, el fin de la vida social y de todo progreso (pp. 2-3).

Desde las funciones sustantivas expresadas en la Misión de la Universidad, según el Estatuto Orgánico (2010): la docencia, la investigación y la proyección social, que deben conducir al estudiante a "aportar soluciones a la problemática y a las necesidades de la sociedad y del país", el Doctorado en Educación hace una apuesta trascendental, en el sentido de que esa función social deberá traspasar las fronteras nacionales y se extenderá también a plantear y a aportar soluciones a la problemática educativa de América Latina y el Caribe, bajo una concepción integralista y universal.

La investigación es la esencia del Doctorado en Educación, de conformidad con lo que establece la Ley 30 de 1992: "los programas de doctorado se concentran en la formación de investigadores a nivel avanzado tomando como base la disposición, capacidad y conocimientos adquiridos por la persona en los niveles 
anteriores de formación. El doctorado debe culminar con una tesis" (artículo 13); por esta razón, las líneas de investigación de este doctorado, que en ese momento surgió con tres líneas: Currículo y Evaluación; Pedagogía, con un énfasis en lo dominicano tomista; y Educación, Derechos Humanos, Política y Ciudadanía, (hoy ya existen dos líneas más: Gestión de la Educación y el Conocimiento y Educación, Sociedad y Cultura), así como las mismas tesis de los doctorandos, constituyen las estrategias fundamentales para alcanzar los objetivos concretos en la búsqueda y solución de las necesidades y los problemas de la educación del país y de América Latina y el Caribe, dentro de un contexto mundial del conocimiento, y antes que buscar el conocimiento por sí mismo, buscar el bienestar y la transformación del ser humano en todas sus dimensiones, así como el desarrollo de la sociedad de una manera sostenible.

Hasta aquí podría decirse que estamos recordando el momento de nacimiento del doctorado. Pero aunque es importante recordar cómo se gestó y cómo nació, es todavía más importante ver cómo se ha desarrollado; y aunque ya han transcurrido cinco años desde que comenzó su vida legal, todavía puede considerarse una criatura incipiente que hasta ahora está aprendiendo a caminar, y así como un niño aprende a caminar cayendo y levantándose, es lógico también que este doctorado no será la excepción. Sin embargo, bien podría pensarse que aprendió a caminar muy rápido y a pasos firmes.

Empecemos desde el momento en que se recibió la aprobación. Ese primer semestre estuvo dedicado a la preparación, en primer lugar, para hacerlo conocer a la comunidad y, en segundo lugar, para recibir, entrevistar y seleccionar a los candidatos que integrarían la primera cohorte, pues no hay duda que el equipo de docentes, bajo la coordinación y dirección de la doctora Marta Osorio, trabajó con mucha dedicación planeando y construyendo todos los documentos protocolares (horarios, plegables, reglamentos, búsqueda y selección de profesores y de directores de tesis, etc.), y preparando la logística necesaria para recibir los primeros 22 candidatos, cuya inauguración se realizó en el Campus de la Universidad con la presencia del Padre Rector, una misa concelebrada y un almuerzo de bienvenida.

Al hacer un balance de estos primero cinco años del doctorado, se puede afirmar con certeza que los resultados son positivos. La acogida por parte de la comunidad educativa no pudo haber sido mejor; si se tiene en cuenta que en las cinco primeras cohortes del doctorado se han matriculado, durante todo este lapso de tiempo, alrededor de 115 aspirantes, cuya deserción ha sido aproximadamente de dos o tres estudiantes por cohorte. Y aunque el desarrollo del programa está 
proyectado para tres años, y dos años más en el caso de no terminar la tesis en esos primeros tres años, hoy el número de egresados ya graduados, tanto de la primera como de la segunda cohorte, es de 15, habiéndose graduado en un promedio de cuatro años.

Hoy, el doctorado dispone de una planta eminente de docentes investigadores nacionales, seis de tiempo completo y de medio tiempo, y cuarenta por orden de prestación de servicios, que dirigen las tesis doctorales y orientan los seminarios de profundización que los doctorandos deben cursar en los primeros tres semestres del programa, todos con título de "Doctor" y algunos con estudios posdoctorales. Sin contar los docentes visitantes que han apoyado estos mismos seminarios, especialmente desde España, México y Argentina, con quienes se ha tenido lazos más estrechos, en aras de los convenios, al tiempo que han aportado una visión mucho más amplia y compleja de la educación iberoamericana. Sin embargo, es preciso recordar que todavía hacen falta más docentes, especialmente de planta, pues el trabajo y las necesidades se multiplican a diario.

Es necesario desatacar también la importancia de los convenios celebrados con varias instituciones nacionales e internacionales, como con la Universidad de San Buenaventura- Seccional de Cali, la Universidad Católica de Manizales, la Universidad Católica de Córdoba (Argentina), la Universidad de Guadalajara (México), el Instituto Católico de París, la Universidad Nacional de Mar del Plata (Argentina), el Ministerio de Educación de la Provincia de Córdoba (Argentina), la Universidad Nacional Pedro Luis Gallo (Perú), la Universidad de Buenos Aires, la Universidad de Granada (España), entre las más importantes. Los convenios han permitido, en primer lugar, hacer visible el doctorado en el extranjero, pues a través de ellos se han hecho intercambios de actividades académicas, sobre todo mediante la participación de los docentes con ponencias en eventos nacionales e internacionales, y se ha logrado que doctores de estas instituciones colaboren con el doctorado en calidad de jurados internacionales de las tesis de los graduandos; en segundo lugar, a los doctorandos se les ha facilitado la realización de sus pasantías, exigidas por el programa antes de recibir la candidatura, acompañados por docentes-doctores de esas mismas instituciones; y en tercer lugar, a través de esos convenios ha tenido cabida el desarrollo de proyectos de investigación interistitucionales e internacionales.

Tampoco se puede dejar pasar por alto las actividades que desde el doctorado se han liderado, tales como investigaciones, publicaciones, organización de eventos y otros. En estos cinco años, además de libros y artículos publicados por cada docente en particular, se han publicado dos volúmenes como recopilación de 
trabajos de los doctorandos de cada cohorte, coordinados por los mismos docentes del doctorado, uno sobre Políticas Educativas y el otro sobre Epistemología y Ciencia; pero están próximos a salir de imprenta el de Pedagogía y el de Gestión de la Educación.

El doctorado ha sido el proponente y ejecutor de dos congresos internacionales: el primero sobre Temas y problemas de la investigación en maestrías, doctorados y posdoctorados en Educación (agosto 17, 18 y 19 de 2011); el segundo, aunque con la participación de toda la Facultad de Educación, sobre Temas y problemas de investigación en educación, sociedad, ciencias y tecnología (septiembre 20 y 21 de 2013). Este año se prepara el tercer congreso sobre temas y problemas de investigación en maestrías y doctorados. En los espacios intermedios de los congresos, se han celebrado dos simposios sobre esta misma temática: el primero de carácter interno y el segundo internacional, en el que ha participado también la Universidad San Buenaventura de Cali; además el doctorado ha estado presente, en forma activa, en otros eventos nacionales e internacionales que sería largo de relatar en este espacio.

De la misma manera, no es posible además, por falta de espacio, referirse a todos los proyectos de investigación en los que ha participado el doctorado, especialmente en las convocatorias internas y externas del Centro de Investigación de la Vicerrectoría de la Universidad Abierta y a Distancia -VUAD- y de la Unidad de Investigación de la Universidad; algunos de estos proyectos ya se han finiquitado con informes y productos: artículos y libros publicados. Otros están todavía en proceso de construcción.

La Revista Interamericana de Investigación, Educativa y Pedagógica RIIEP ha sido indexada y reindexada, en categoría $C$, gracias a los esfuerzos de los doctores José Arlés Gómez y Edgar Mauricio Martínez, que han estado al frente; asimismo, es preciso destacar la creación y conformación de la Red de Doctorados en Educación de las universidades privadas, trabajo que se comenzó a realizar inicialmente con la doctora Marta Osorio como directora del doctorado, en el momento está al frente de este proyecto la doctora Claudia Vélez. Por último, se destacaría también el programa de estudios posdoctorales en Educación, Ciencias Sociales e Interculturalidad, que nació y ha sido coordinado por el director del doctorado, doctor José Arlés Gómez. En la primera cohorte de posdoctorado recibieron su certificación en estudios posdoctorales un número de 13 doctores nacionales y extranjeros, entre ellos, tres de la República del Perú, y cuyos trabajos investigativos están también en proceso de publicación como libro colectivo. 
Se está conformando la segunda cohorte que próximamente iniciará los estudios en este mismo nivel.

Quizás se queden muchas cosas por fuera de esta semblanza; sin embargo, resta todavía la pregunta: ¿qué falta todavía por hacer? Sin duda muchas cosas. Me permitiré una metáfora comparando el doctorado con un organismo vivo que recibe el influjo de su entorno. Todo organismo vivo es un sistema complejo abierto que intercambia energía positiva y negativa con su medio, fenómeno que en física se conoce con el nombre de entropía; una parte de esa energía infortunadamente se pierde y no se puede volver a recuperar para producir nuevo trabajo; pero el sistema, al intercambiar energía negativa, recibe también aportaciones positivas del medio, y como todo cuerpo vivo que recibe alimento, luz, aire, agua para poder mantenerse, así el doctorado necesita más interacción con otras instituciones y programas análogos del orden nacional e internacional. Es necesario trabajar para que se reconozca, cada vez más, no solamente a través de los sistemas publicitarios, sino más que todo por la calidad de sus egresados y la calidad de la producción de conocimientos científicos y filosóficos en los diferentes campos de la educación y la pedagogía, propios de su competencia.

El doctorado tendrá que trabajar con mayor ahínco para crear doctrina y hacer escuela en el campo de las ciencias de la educación y la pedagogía, con impacto e incidencia efectiva en la transformación de los macro-problemas educativos nacionales y de América Latina y el Caribe, hacia donde, especialmente, ha sido proyectado el doctorado desde sus inicios. Pero tampoco se puede pretender que un doctorado resuelva todos los problemas, pues para eso están las voluntades políticas y tecnocráticas a los que les compete y son los directos responsables de llevar a la práctica las ideas de quienes las proponen. El papel de un doctorado debe ser el de pensar y repensar la educación, aportando soluciones con sus ideas, como bien lo recordaba el doctor Guillermo Hoyos, (Q.E.P.D), pues, las ideas son las que mueven el mundo. Por esta razón, es necesario dedicarle la mayor atención a las tesis doctorales, procurando que estas sean de la altura y de la profundidad que requiere el nivel doctoral, para que no terminen como simples descripciones de la realidad o de los problemas, repitiendo el conocimiento en boga, con muy poco o, algunas veces, sin ningún aporte científico, filosófico y social a la problemática educativa.

Por otra parte, hay que buscar mecanismos para que los convenios con instituciones nacionales y extranjeras funcionen realmente y no se queden escritos en un documento sin vida. No se necesitan muchos convenios; pueden ser unos pocos, pero que se hagan efectivos mediante procesos de intercambio recíproco: 
estudiantes, docentes, investigaciones, publicaciones conjuntas, participación en eventos, entre los más importantes. El doctorado recibe ya bastante de otras latitudes; pero de aquí, todavía se llevan muy pocas cosas. La educación colombiana y este doctorado tienen bastantes cosas para mostrar y aportar, tanto en cuanto a experiencia como cognoscitivamente, a otros países, especialmente del continente. Esto debe ser parte del proceso de internacionalización.

Un trabajo que falta por hacer es el de fortalecer, cada vez más, los grupos de investigación del doctorado. Se necesita buscar la empatía con personas, docentes y estudiantes, que quieran trabajar en equipo y que aporten con sus productos a los grupos y al doctorado, para que cada miembro del grupo no funcione como rueda suelta. Colciencias valora mucho la cohesión de los grupos.

\section{Conclusiones}

Para terminar esta intervención conviene solicitar, de manera muy especial, a los cuadros directivos, que no miren los doctorados como un programa más de la Universidad al que se le exige que muestre resultados, pero con demasiadas restricciones, especialmente en el orden económico. Los doctorados, por lo general, no producen ganancia en dinero. La ganancia está en el prestigio y la excelencia de lo que producen en lo profesional, científico y académico. La Universidad todavía no tiene muy claras y precisas las políticas con respecto a los doctorados. Un doctorado no puede medirse con los mismos parámetros que se mide un pregrado o inclusive cualquier otro programa de posgrado. Los doctorados tienen dinámicas muy diferentes; su esencia es la investigación y la producción de conocimiento; pero es necesario que la universidad conozca y reconozca cuáles son esas dinámicas para que trace las políticas adecuadas, ya que muchas cosas de las que se han hecho en el doctorado han sido resultado más de la experiencia y de un aprendizaje que se ha realizado en el camino, por la falta de directrices válidas para este nivel de formación. Algunas veces se tiene la impresión de que las únicas directrices para el doctorado, emanadas de las instancias de dirección y gestión, se reducen al Ileno de formalidades documentales que, aunque son necesarias, distraen, con frecuencia, en el trabajo fundamental del doctorado, es decir, en la investigación y la producción de conocimiento.

Al estar ad portas de renovar registro, pues queda prácticamente menos de un año para la entrega del documento de renovación, el compromiso no es solamente 
del equipo que está al frente del doctorado, sino de toda la Universidad, de la Vicerrectoría, de la Facultad de Educación, de los egresados, de los doctorandos activos $\mathrm{y}$, en general, de la comunidad educativa; solo de esta manera será posible cumplir con todos y cada uno de los requisitos que se necesitan para la renovación que se aproxima, y una vez obtenida, pensar enseguida en el registro de alta calidad.

\section{Referencias}

Almeida, E. (2003, septiembre). Prospetiva di Tomasso D’Aquino. Ponencia en el Congresso Tomista Internazionale. L'Umanismo Cristiano nel III Millenio. Roma: Pontificia Accademia di San Tommaso-Societ Internazionale Tommaso d'Aquino. Recuperado de http://e-aquinas.net/pdf/almeida/pdf

Departamento Nacional de Planeación. (2006). Plan Nacional de Desarrollo 2006-2010. Bogotá, Colombia. Recuperado de www.dnp.gov.co

Ministerio de Educación Nacional. (2006). Plan Decenal de Educación 2006-2016. Bogotá, Colombia. Recuperado de www.plandecenal.edu.co

República de Colombia. (1992). Ley 30 de 1992. Bogotá.

Universidad Santo Tomás. (2002). Estatuto Orgánico. Bogotá: USTA.

Universidad Santo Tomás. (2009). Doctorado en Educación. Documento Marco. Bogotá. 